\title{
An Open-Label, Intra-Individual Study to Evaluate a Regimen of Three Cosmetic Products Combined with Medical Treatment of Rosacea: Cutaneous Tolerability and Effect on Hydration
}

Francine Santoro · Nadège Lachmann

Received: July 26, 2019 / Published online: October 17, 2019

(C) The Author(s) 2019

\section{ABSTRACT}

Introduction: Although rosacea management includes general skincare, previous studies have not evaluated comprehensive skincare regimens as adjuvants to other treatments.

Methods: The primary objective of this openlabel, intra-individual study of subjects with rosacea was to evaluate the cutaneous tolerability of a regimen consisting of Cetaphil PRO Redness Control Day Moisturizing Cream (once daily in the morning), Cetaphil PRO Redness Control Night Repair Cream (once daily in the evening) and Cetaphil PRO Redness Control Facial Wash (foam once in the morning and once in the evening). Secondary objectives were to evaluate the effect on transepidermal water loss (TEWL) and cutaneous hydration and to determine the subjects' evaluation of efficacy, tolerability and future use. A dermatologist examined subjects and measured TEWL and cutaneous hydration on day (D) 0, D7 and D21, when subjects ranked symptoms. Subjects completed a questionnaire on D21.

Results: The per-protocol population consisted of 42 subjects receiving treatment for rosacea.

Enhanced Digital Features To view enhanced digital features for this article go to https://doi.org/10.6084/ m9.figshare.9901295.

F. Santoro $(\bowtie) \cdot$ N. Lachmann

Galderma SA, Lausanne, Switzerland

e-mail: francine.santoro@galderma.com
Eleven subjects developed adverse events, none of which were considered to be related to the skincare products. Five subjects showed signs or symptoms that were potentially associated with the skincare products that might suggest poor cutaneous tolerability; these were generally mild. TEWL decreased significantly by a mean of $17 \%$ on D7 and a mean of $28 \%$ on D21 compared with baseline (both $P<0.001$ ). Skin hydration increased significantly by a mean of $5 \%$ on D7 $(P=0.008)$ and a mean of $10 \%$ on D21 $(P<0.001)$ compared with baseline. Subjects reported that the regimen was pleasant (98\%) and effective (95\%) and that it offered various benefits; $90 \%$ of subjects reported that they would like to continue to use the regimen and would buy the products.

Conclusion: The skincare regimen improved skin hydration and skin barrier function in subjects receiving medical treatment for rosacea and was well tolerated.

Funding: Galderma S.A.

Keywords: Moisturizing cream; Rosacea; Skincare regimen

\section{INTRODUCTION}

Approximately $5-10 \%$ of the population suffers from rosacea, a chronic inflammatory skin disorder [1, 2], which can have a marked psychological impact and undermine health-related 
quality of life [3-6]. Treatment depends on the phenotype and is supported by general skincare, which includes using sunscreen, gentle cleansers and frequent application of moisturizers [7]. According to recently published treatment guidelines, general skincare is also the main strategy to manage secondary features, such as dry appearance and dry and stinging sensations [7].

In people with rosacea, moisturizers can repair and maintain barrier function, enhance skin hydration, reduce the likelihood of skin irritation, relieve dry skin and improve dermatological characteristics (such as softness and suppleness) [8]. Skin care products for people with rosacea should block ultraviolet (UV) light; sun exposure is reported as a contributory factor by $61-81 \%$ of rosacea subjects $[3,9]$.

People with rosacea, however, often experience dry facial skin that can exacerbate symptoms [8] as well as heightened skin sensitivity [10]. For example, in a study of 65 people with rosacea and 60 healthy subjects, 30.8 and $10.0 \%$, respectively, showed a positive reaction to at least one item in the 57-item Chemotechnique Diagnostics Cosmetic Series [11]. The reactive skin characteristic of rosacea seems to arise from activation of cutaneous nerves, a dysfunctional innate immune response and impaired epidermal barrier function [11].

As a result, skin care regimens should be assessed in people with rosacea. While moisturizers can be combined with other systemic and topical rosacea therapies [8], so far as we are aware, no previous studies have evaluated a comprehensive skincare regimen consisting of more than one product as an adjuvant to medical treatment of rosacea. This is, therefore, the first study to evaluate the cutaneous tolerability and effect on hydration and skin barrier function of a regimen of three cosmetic productsCetaphil PRO Redness Control Day Moisturizing Cream (sun protection factor 30), Cetaphil PRO Redness Control Night Repair Cream and Cetaphil PRO Redness Control Facial Wash (all Galderma S.A., Lausanne, Switzerland)—during 21 days of use in subjects receiving concomitant medical treatment for rosacea. We hypothesized that the use of a comprehensive skincare regimen, as an adjuvant to medical treatment, would improve the skin characteristics of patients with rosacea.

\section{METHODS}

\section{Study Design}

This open-label, intra-individual study was performed by Dermscan Poland (Gdansk, Poland). Measurements and evaluations were taken before and after application of the regimen. Therefore, subjects were their own controls.

Subjects applied Cetaphil PRO Redness Control Facial Wash ('wash foam') once in the morning and once in the evening, the Cetaphil PRO Redness Control Day Moisturizing Cream ('sunscreen cream'), a beige emulsion, once daily in the morning and the Cetaphil PRO Redness Control Night Repair Cream ('night cream'), a white emulsion, once daily in the evening. Subjects applied the regimen for 21 days to their face. Patients were asked not to apply any product on the evening before each study visit. Washing with water was allowed.

\section{Inclusion and Exclusion Criteria}

The study aimed to enroll at least 40 female or male subjects, aged between 25 and 75 years, with Type I or Type II rosacea presenting with mild to moderate non-transient erythema. The skin color of the subjects was classified as ranging from Fitzpatrick phototype I to IV. A maximum of $20 \%$ of subjects were older than 60 years. Subjects received an oral or topical rosacea treatment for at least 4 weeks before enrollment. Subjects were not permitted to change their rosacea treatment in the 4 weeks before enrollment and were otherwise healthy. Female subjects of childbearing potential were asked to use precautions to avoid pregnancy.

Exclusion criteria included: starting, stopping or changing hormonal treatment (including contraception) during the 3 months before and during the study; a history of skin cancer; vaccination during the previous 2 weeks or intending to be vaccinated during the study; 
and active skin disease (except rosacea) at the test area.

Subjects were also excluded if they used topical medication or over-the-counter treatments on the test area (excluding rosacea treatment), systemic immuno-suppressive drugs or antihistamines in the 4 weeks before or during the study. Subjects were excluded if they took anti-inflammatories and analgesics (except for minor pain relief medicine, such as acetylsalicylic acid or paracetamol) within 3 days of the start of study, reported excessive sun exposure 2 weeks before or during the study, or underwent procedures such as laser treatment or electrocoagulation in the 6 weeks before or during the study. Subjects with a history of adverse reactions to cosmetics or other common triggers were also excluded.

\section{Objectives}

The primary objective was to evaluate the cutaneous tolerability of a skincare regimen consisting of a wash foam, daily sunscreen cream and night cream after 21 days as an adjuvant to medical treatment for rosacea. The secondary objectives were to evaluate the effect of the skincare regimen on transepidermal water loss (TEWL) and cutaneous hydration, and to determine subjects' subjective evaluation of the efficacy, tolerability and their likely future use of the skincare regimen.

\section{Cutaneous Tolerability}

The same dermatologist examined each subject's face on day (D) 0, D7 and D21 and ranked each of the following using a five-point scale: erythema; edema; desquamation and dryness; roughness; other signs (Table 1 ). In the latter case, the dermatologist specified the sign (e.g., telangiectasia, papules, and pustules). On D7 and D21, the dermatologist evaluated global tolerability (defined as the least favorable result) based on the clinical examination and subject reports. The dermatologist assessed itching, irritation and subjective rosacea symptoms by asking the patient.
On D0, D7 and D21 subjects ranked each of the following using a five-point scale (0: none; 1: very mild; 2 : mild; 3 : moderate; 4 : severe): tightness; stinging; itching; warm or burning sensation; dryness; other. Again, the subject specified any other signs.

\section{TEWL and Cutaneous Hydration}

Transepidermal water loss was measured using a Tewameter ${ }^{\circledR}$ TM 300 measuring device (Courage \& Khazaka $\mathrm{GmbH}$, Cologne, Germany) on D0, D7 and D21 after 30 min of acclimatization in ambient conditions (temperature $22 \pm 2{ }^{\circ} \mathrm{C}$, relative humidity of between 35 and 55\%). Measurements were taken in one area on the left or right jaw line (alternating sides between consecutive subjects).

Measurements of cutaneous hydration were performed using a Corneometer ${ }^{\circledR}$ CM 825 skin hydration measurement instrument (Courage \& Khazaka GmbH) on D0, D7 and D21 after 30 min of acclimatization in ambient conditions (see above) on the same facial area as the TEWL measurement. The same area was used throughout the study for both measurements.

\section{Subjects' Subjective Evaluation}

Subjects completed a questionnaire on D21 asking for their opinions about the efficacy, tolerability and their likely future use of the skincare regimen.

\section{Ethical Approval}

The protocol was approved by the Internal Ethic Committee at Dermscan Poland. The study was conducted according to Good Clinical Practice and the Declaration of Helsinki (1964) and its successive updates were followed. Informed consent to participate was obtained from all participants. According to cosmetic regulations (as all products tested in this study were cosmetics rather than drugs), the study did not require registration. 
Table 1 Scale for dermatologist's assessment of cutaneous tolerability

\begin{tabular}{|c|c|c|c|c|c|}
\hline \multirow[t]{2}{*}{ Outcome } & \multicolumn{5}{|c|}{ Severity (score) } \\
\hline & None (0) & Very mild (1) & Mild (2) & Moderate (3) & Severe (4) \\
\hline Erythema & Not present & $\begin{array}{l}\text { Fairly } \\
\text { detectable, } \\
\text { discreet } \\
\text { pinkness }\end{array}$ & Definite pinkness & $\begin{array}{l}\text { Clearly distinguishable, } \\
\text { dull red }\end{array}$ & $\begin{array}{l}\text { Deep dark or fiery } \\
\text { bright red }\end{array}$ \\
\hline Edema & Not present & $\begin{array}{l}\text { Palpable, barely } \\
\text { visible }\end{array}$ & Palpable and visible & $\begin{array}{l}\text { Obvious edema } \\
\text { (thickness }<1 \mathrm{~mm}) \text {, } \\
\text { with or without } \\
\text { papule(s) or vesicle(s) }\end{array}$ & $\begin{array}{l}\text { Severe edema } \\
\text { (thickness }>1 \mathrm{~mm} \text { ), } \\
\text { with or without } \\
\text { vesicle }(\mathrm{s}) \text { or blister(s) }\end{array}$ \\
\hline $\begin{array}{l}\text { Desquamation/ } \\
\text { dryness }\end{array}$ & $\begin{array}{l}\text { Normal } \\
\text { skin, } \\
\text { regular } \\
\text { cutaneous } \\
\text { surface, } \\
\text { absence of } \\
\text { scale }\end{array}$ & $\begin{array}{l}\text { Cutaneous } \\
\text { surface } \\
\text { slightly dull, } \\
\text { little scales } \\
\text { only with } \\
\text { light } \\
\text { scratching or } \\
\text { rubbing }\end{array}$ & $\begin{array}{l}\text { More obvious scales } \\
\text { with a slightly } \\
\text { whitish aspect }\end{array}$ & $\begin{array}{l}\text { Numerous marked } \\
\text { scales }\end{array}$ & Heavy scale production \\
\hline Roughness & $\begin{array}{l}\text { Surface } \\
\text { perfectly } \\
\text { smooth } \\
\text { and supple }\end{array}$ & $\begin{array}{l}\text { Slight } \\
\text { irregularity } \\
\text { and mild } \\
\text { roughness to } \\
\text { the tangential } \\
\text { touch }\end{array}$ & $\begin{array}{l}\text { Markedly irregular } \\
\text { and rough } \\
\text { appearance and } \\
\text { ability to feel by } \\
\text { vertical touch } \\
\text { slight skin } \\
\text { roughness }\end{array}$ & $\begin{array}{l}\text { Very marked feeling of } \\
\text { roughness and } \\
\text { irregularity }\end{array}$ & $\begin{array}{l}\text { Presence of very } \\
\text { important } \\
\text { irregularities, } \\
\text { dehydrated sensation }\end{array}$ \\
\hline Other signs & None & Very mild & Mild & Moderate & Severe \\
\hline
\end{tabular}

\section{Statistical Analysis}

Paired Student $t$ tests compared TEWL and cutaneous hydration values before, during and after treatment. A $P$ value of $\leq 0.05$ was considered to be statistically significant. Statistical analyses were performed using Excel 2010 (Microsoft Corp., Redmond, WA, USA). Based on the bilateral confidence interval of a proportion, a more than $65.4 \%$ agreement in responses to questions in the subjects' subjective evaluation was considered to be statistically significant.

\section{RESULTS}

\section{Subject Disposition and Demographics}

A total of 44 subjects were enrolled, of whom 42 (37 females; mean age 48 years; range 25-68 years) competed the study (Table 2). Eighteen patients had type 1 rosacea; the remainder had type 2 rosacea. All subjects received medical treatment for rosacea for at least 30 days before enrollment in the study. Of these, 20 subjects received more than one medical treatment for rosacea (median 1; range 1-4). The 
Table 2 Demographics of the per-protocol population $(n=42)$

\begin{tabular}{|c|c|}
\hline Variable & Value \\
\hline \multicolumn{2}{|l|}{ Age (years) } \\
\hline Mean & 48 \\
\hline Median & 25 \\
\hline Range & $25-68$ \\
\hline \multicolumn{2}{|l|}{$\operatorname{Sex}(n)$} \\
\hline Female & 37 \\
\hline Male & 5 \\
\hline \multicolumn{2}{|l|}{ Fitzpatrick phototype $(n)$} \\
\hline I & 6 \\
\hline II & 22 \\
\hline III & 12 \\
\hline IV & 2 \\
\hline \multicolumn{2}{|l|}{ Facial skin type $(n)$} \\
\hline Dry & 20 \\
\hline Normal & 6 \\
\hline Combination & 14 \\
\hline Greasy & 2 \\
\hline \multicolumn{2}{|l|}{ Rosacea type } \\
\hline I & 18 \\
\hline II & 24 \\
\hline \multicolumn{2}{|l|}{ Medical treatments for rosacea $(n)^{\mathrm{a}}$} \\
\hline Metronidazole & 32 \\
\hline Ivermectin & 13 \\
\hline Clindamycin & 5 \\
\hline Azelaic acid & 4 \\
\hline Lymecycline & 3 \\
\hline Tacrolimus & 3 \\
\hline Erythromycin & 2 \\
\hline Adapalene plus benzoyl peroxide & 1 \\
\hline Azelaic acid plus lactobionic acid & 1 \\
\hline Benzoyl peroxide & 1 \\
\hline Brimonidine & 1 \\
\hline
\end{tabular}

Table 2 continued

\begin{tabular}{ll}
\hline Variable & Value \\
\hline Clindamycin plus tretinoin & 1 \\
Clotrimazole & 1 \\
Niverosin $^{\mathrm{b}}$ & 1 \\
\hline
\end{tabular}

a Subjects could receive more than one treatment

b Niverosin: branded combination of diosmin, rutoside and hesperidin

safety population consisted of 44 subjects who applied the study products at least once. Of these, 42 subjects constituted the per-protocol population, who were analyzed for cutaneous tolerability and completed the questionnaire.

One subject was lost to follow-up after D7 and was excluded from the analysis. One subject withdrew on D7 after developing the following symptoms: moderate erythema on the cheeks, nose and chin; moderate desquamation, dryness and roughness on the whole face; and reporting a severe warm, burning sensation on the cheeks. This subject had changed rosacea treatment 1 week before the start of the study, but neglected to inform the investigator. Due to this serious protocol violation, which made assessing causality with the study products impossible, the subject was excluded. This was the only serious protocol violation.

Values for TEWL were recorded for 39 subjects on D7 and 42 subjects on D21. Cutaneous hydration was recorded for 41 subjects on D7 and D21. The other values were deemed aberrant due to technical issues during measurement based on the investigator's experience (values did not correlate with variations in skin hydration or clinical examination) and, therefore, excluded.

\section{Adverse Events and Cutaneous Tolerability}

Twelve subjects reported a total of 15 adverse events, most commonly colds or flu $(n=4)$, 
runny nose $(n=3)$ and headache $(n=2)$. None of the adverse events were considered to be related to the skincare products. No serious adverse events were observed. Five subjects showed signs or symptoms that might suggest poor cutaneous tolerability; these were potentially associated with the skincare products (Table 3). These symptoms were generally mild. In addition, based on the subjective evaluation questionnaire, $26 \%$ of subjects reported cutaneous irritation during the study.

\section{TEWL and Cutaneous Hydration}

Transepidermal water loss decreased significantly by a mean of $17 \%$ on D7 and a mean of $28 \%$ on D21 compared with baseline (both $P<0.001$ ) (Table 4; Fig. 1). Hydration of the superficial layers of the epidermis increased significantly by a mean of $5 \%$ on D7 $(P=0.008)$ and a mean of $10 \%$ on D21 $(P<0.001)$ compared with baseline (Table 5; Fig. 2).

\section{Subjects' Subjective Evaluation}

Subjects reported that the skincare regimen was pleasant (98\%) and effective (95\%). The questionnaire results suggested a range of benefits on, among other outcomes, dryness, moisturizing and stinging (Table 6). Subjects found the skincare products to be gentle and appropriate for use with medical treatments for rosacea (Table 6). Indeed, 90\% of subjects reported that they would like to continue to use the skincare regimen; the same proportion would like to buy the products, regardless of the price. All results exceeded $65.4 \%$ agreement and are, therefore, statistically significant.

\section{DISCUSSION}

A skincare regimen encompassing sunscreen, gentle cleansers and frequent application of moisturizers is a cornerstone of rosacea management [7]. The combination of the three

Table 3 Symptoms that might suggest poor cutaneous tolerability and potential association with the skincare regimen

\begin{tabular}{|c|c|c|}
\hline $\begin{array}{l}\text { Subject } \\
\text { number }\end{array}$ & $\begin{array}{l}\text { Study } \\
\text { period }\end{array}$ & Adverse event \\
\hline 28 & D1-D12 & $\begin{array}{l}\text { Subject reported moderate tightness on the cheeks, which lasted } 14 \mathrm{~h} \text {, beginning immediately } \\
\text { after application of the day cream }\end{array}$ \\
\hline 30 & D14-D21 & $\begin{array}{l}\text { Subject reported moderate tightness and dryness on the whole face, which lasted } 1 \mathrm{~h} \text {, beginning } \\
\text { immediately after application of the day cream }\end{array}$ \\
\hline \multirow[t]{3}{*}{38} & D1-D6 & $\begin{array}{l}\text { Subject reported severe tightness on the cheeks and chin, which lasted a few hours, beginning } \\
1 \mathrm{~h} \text { after application of the day cream }\end{array}$ \\
\hline & D7-D20 & $\begin{array}{l}\text { Subject reported mild tightness on the cheeks and chin, which lasted } 3 \mathrm{~h} \text {, beginning } 1 \mathrm{~h} \text { after } \\
\text { application of the day cream }\end{array}$ \\
\hline & $\mathrm{D} 21$ & Dermatologist observed very mild desquamation and dryness on the chin \\
\hline 39 & $\mathrm{D} 21$ & Dermatologist observed very mild desquamation and dryness on the cheeks and chin \\
\hline \multirow[t]{3}{*}{41} & D0-D6 & $\begin{array}{l}\text { Subject reported mild tightness on the nose, cheeks and forehead, which lasted the whole day, } \\
\text { beginning } 5 \mathrm{~min} \text { after application of the day cream }\end{array}$ \\
\hline & $\begin{array}{l}\text { D5 and } \\
\text { D6 }\end{array}$ & $\begin{array}{l}\text { Subject reported moderate burning sensation on the nose, which lasted } 1-2 \mathrm{~h} \text {, beginning } 5 \mathrm{~min} \\
\text { after application of the day cream }\end{array}$ \\
\hline & D21 & Dermatologist observed very mild desquamation and dryness, and roughness on the nose \\
\hline
\end{tabular}

$D$ Day 
Table 4 Transepidermal water loss

\begin{tabular}{lclll}
\hline Study period & $\begin{array}{l}\text { Mean } \pm \text { SEM } \\
\left(\mathbf{g ~ m}^{-2} \mathbf{h}^{-1}\right)\end{array}$ & Change (\%) & $\boldsymbol{P}$ value & $\begin{array}{l}\text { Subjects with improved } \\
\text { TEWL (\%) }\end{array}$ \\
\hline D0 & $13.4 \pm 0.7$ & & \\
D7 & $11.1 \pm 0.5$ & & \\
D21 & $9.7 \pm 0.5$ & & \\
$\Delta$ D7-D0 & $-1.8 \pm 0.4$ & -17 & $<0.001$ & 74 \\
$\Delta$ D21-D0 & $-3.7 \pm 0.5$ & -28 & $<0.001$ & 93 \\
$\Delta$ D21-D7 & $-1.8 \pm 0.4$ & -13 & $<0.001$ & 82 \\
\hline
\end{tabular}

$\triangle$ Change, SEM standard error of the mean, TEWL transepidermal water loss

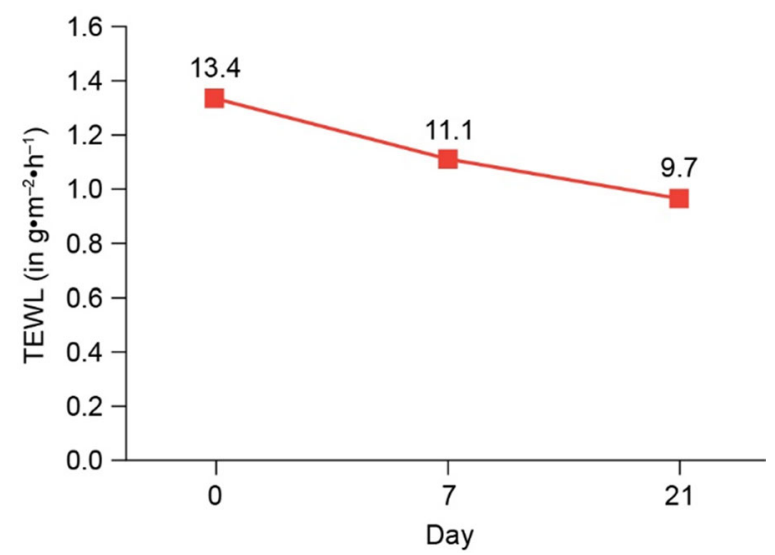

Fig. 1 Mean transepidermal water loss $(T E W L)$ over time

Table 5 Cutaneous hydration

\begin{tabular}{lllr}
\hline Study period & $\begin{array}{l}\text { Mean } \pm \text { SEM } \\
(\mathrm{AU})\end{array}$ & $\begin{array}{l}\text { Change } \\
(\%)\end{array}$ & $\boldsymbol{P}$ value \\
\hline D0 & $56 \pm 2$ & \\
D7 & $59 \pm 2$ & \\
D21 & $61 \pm 2$ & & \\
$\Delta$ D7-D0 & $3 \pm 1$ & 5 & 0.008 \\
$\Delta$ D21-D0 & $6 \pm 1$ & 10 & $<0.001$ \\
$\Delta$ D21-D7 & $3 \pm 1$ & 4 & 0.003 \\
\hline
\end{tabular}

$\triangle$ Change, SEM standard error of the mean, $A U$ Arbitrary units

products assessed in this study (wash foam, daily sunscreen cream and night cream) meet these needs. Despite many people with rosacea reporting heightened skin sensitivity with skin care and personal hygiene products $[10,11]$, to the best of our knowledge, no previous studies have evaluated a comprehensive skincare regimen as adjuvant to the medical treatment of rosacea. This is, therefore, the first study to evaluate the cutaneous tolerability and effect on hydration and skin barrier function of a regimen of three cosmetic products in subjects receiving concomitant medical treatments for rosacea.

Despite the subjects having heightened skin sensitivity $[10,11]$, the skincare regimen assessed here (Cetaphil PRO Redness Control Day Moisturizing Cream, Cetaphil PRO Redness Control Night Repair Cream and Cetaphil PRO Redness Control Facial Wash) showed good cutaneous tolerance when used daily with concurrent topical or systemic treatments for rosacea for 21 days. While a few subjects reported signs and symptoms that might indicate poor cutaneous tolerability, these were generally mild and usually related to the day cream. However, as subjects used a variety of medical treatments, assessing causality is difficult. Moreover, $26 \%$ of subjects reported cutaneous irritation during the study. The questionnaire did not enquire about the severity of the irritation. Moreover, $12 \%$ of subjects showed signs or symptoms (most of which were mild) that might suggest poor cutaneous tolerability, with these signs and symptoms potentially associated with the skincare products. However, 98\% of subjects reported that using the skincare regimen was pleasant. The high level of 


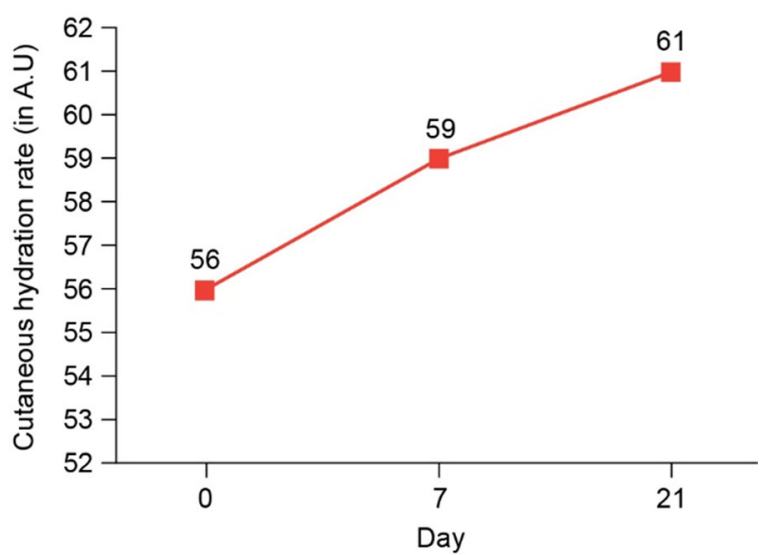

Fig. 2 Mean cutaneous hydration over time. $A U$ Arbitrary units

acceptance suggests that the irritation was not sufficiently serious to influence the subjects' perception of the product.

Moisturizers relieve dry skin, improve dermatological characteristics, such as softness and suppleness, and repair defective barrier function [8]. Despite subjects receiving concurrent topical or systemic treatments for rosacea, 21 days use of the skincare regimen significantly reduced TEWL and increased cutaneous hydration. These results suggest that the skincare regimen repaired the cutaneous barrier in rosacea subjects over and above any effect of the concurrent topical or systemic treatments.

TEWL and cutaneous hydration improved significantly on D21 compared with D7, and prolonged use of this skincare regimen may further improve outcomes. Studies with longerterm follow-up could ascertain whether and for how long the cutaneous barrier continues to repair.

Ninety percent of subjects valued the skincare regimen and, tellingly, wanted to continue use after the study ended. A product that is well accepted is likely to help ensure good adherence, especially during long-term use, although further studies with longer follow-up are needed.

The study was open label, un-randomized and performed at one center. Therefore, this study could be replicated in a larger, more

Table 6 Subjects' subjective evaluation of efficacy of the skincare regimen after D21

\begin{tabular}{|c|c|c|c|}
\hline Characteristic & $\begin{array}{l}\text { Percentage of subjects } \\
\text { who fully agree or agree }\end{array}$ & $\begin{array}{l}\text { Percentage of } \\
\text { subjects who fully } \\
\text { agree }\end{array}$ & $\begin{array}{l}\text { Percentage of } \\
\text { subjects who } \\
\text { agree }\end{array}$ \\
\hline Protects the skin from drying out & 92 & 40 & 52 \\
\hline Provides long-lasting moisturization and relief & 93 & 43 & 50 \\
\hline $\begin{array}{l}\text { Safe to use in combination with systemic or } \\
\text { topical drugs for rosacea }\end{array}$ & 100 & 60 & 40 \\
\hline $\begin{array}{l}\text { Decreases symptoms associated with dry } \\
\text { appearance, dry sensation, stinging sensation }\end{array}$ & 93 & 50 & 43 \\
\hline $\begin{array}{l}\text { Complimentary for use with redness and/or } \\
\text { rosacea treatments }\end{array}$ & 86 & 36 & 50 \\
\hline Non-irritating for redness-prone skin & 100 & 52 & 48 \\
\hline Gentle enough for everyday use & 98 & 60 & 38 \\
\hline $\begin{array}{l}\text { Helps alleviate dryness associated with rosacea } \\
\text { treatments }\end{array}$ & 92 & 52 & 40 \\
\hline $\begin{array}{l}\text { Leaves skin feeling moisturized and } \\
\text { comfortable }\end{array}$ & 93 & 50 & 43 \\
\hline
\end{tabular}


diverse, randomized, double-blind sample. Moreover, future studies could determine any interactions between the medical treatments and the skincare regimen and use biometric outcomes to characterize skin condition in addition to the subjective evaluations by patients and experienced physicians.

Furthermore, the population was not homogenous in terms of demographics, disease severity or drug treatment. However, a highly homogenous population may reduce the external validity of the study. Our study represents the wider, demographically diverse population of subjects with rosacea who, in clinical practice, receive a range of treatments. Despite this variation, the regimen improved skin hydration and barrier function, suggesting broad clinical applicability. The study could be repeated, however, in a population selected by more tightly defined inclusion and exclusion criteria, such as infestation with Demodex mites. Subjects with rosacea show a significantly higher prevalence (odds ratio 9.0) of infestation and density of Demodex mites (standardized mean difference 1.6) compared with control patients. A causal relationship has not, however, been established [12].

\section{CONCLUSIONS}

The skincare regimen of Cetaphil PRO Redness Control Day Moisturizing Cream, Cetaphil PRO Redness Control Night Repair Cream and Cetaphil PRO Redness Control Facial Wash improved skin hydration and barrier function in subjects who were receiving medical treatment for rosacea. Subjects reported that the skincare regimen was pleasant, effective and offered a range of benefits. Considering the skin reactivity of rosacea subjects, the skincare regimen's tolerability was extremely favorable, underscored by the fact that most participants reported that they would purchase and recommend the products. These findings suggest that this skincare regimen could improve outcomes for people receiving medical treatments for rosacea.

\section{ACKNOWLEDGEMENTS}

The authors thank the study participants for their involvement in this study.

Funding. Sponsorship for this study and article processing charges were funded by Galderma SA to support the work on this project. All authors had full access to all of the data in this study and take complete responsibility for the integrity of the data and accuracy of the data analysis.

Editorial Assistance. Editorial assistance in the preparation of this article was provided by Mark Greener and Marian East of MedSense Ltd. Support for this assistance was funded by Galderma SA.

Authorship. All named authors meet the International Committee of Medical Journal Editors (ICMJE) criteria for authorship for this article, take responsibility for the integrity of the work as a whole, and have given their approval for this version to be published.

Disclosures. Francine Santoro is an employee of Galderma SA. Nadège is an employee of Galderma SA.

Compliance with Ethics Guidelines. The protocol was approved by the Internal Ethic Committee at Dermscan Poland. The study was conducted according to Good Clinical Practice and the Declaration of Helsinki (1964) and its successive updates were followed. Informed consent to participate was obtained from all participants. According to cosmetic regulations (as all products tested in this study were cosmetics rather than drugs), the study did not require registration.

Data Availability. The datasets generated during and/or analyzed during the current study are not publicly available because they are commercially sensitive, but are available from the corresponding author on reasonable request. 
Open Access. This article is distributed under the terms of the Creative Commons Attribution-NonCommercial 4.0 International License (http://creativecommons.org/licenses/ by-nc/4.0/), which permits any noncommercial use, distribution, and reproduction in any medium, provided you give appropriate credit to the original author(s) and the source, provide a link to the Creative Commons license, and indicate if changes were made.

\section{REFERENCES}

1. Wehausen B, Hill DE, Feldman SR. Most people with psoriasis or rosacea are not being treated: A large population study. Dermatol Online J. 2016;22(7).

2. Abokwidir M, Feldman SR. Rosacea management. Skin Appendage Disord. 2016;2:26-34.

3. Blount B, Pelletier A. Rosacea: a common, yet commonly overlooked, condition. Am Fam Physician. 2002;66:435-40.

4. Moustafa F, Lewallen RS, Feldman SR. The psychological impact of rosacea and the influence of current management options. J Am Acad Dermatol. 2014;71:973-80.

5. Bewley A, Fowler J, Schöfer H, et al. Erythema of rosacea impairs health-related quality of life: results of a meta-analysis. Dermatol Ther. 2016;6:237-47.
6. van der Linden MM, van Rappard DC, Daams JG, et al. Health-related quality of life in patients with cutaneous rosacea: a systematic review. Acta Derm Venereol. 2015;95:395-400.

7. Schaller M, Almeida LMC, Bewley A, et al. Rosacea treatment update: recommendations from the global ROSacea COnsensus (ROSCO) panel. Br J Dermatol. 2017;176:465-71.

8. Draelos ZD, Ertel K, Berge C. Niacinamide-containing facial moisturizer improves skin barrier and benefits subjects with rosacea. Cutis. 2005;76:13541 .

9. Oge LK, Muncie HL, Phillips-Savoy AR. Rosacea: diagnosis and treatment. Am Fam Physician. 2015;92:187-96.

10. Del Rosso JQ, Thiboutot D, Gallo R, et al. Consensus recommendations from the American Acne \& Rosacea Society on the management of rosacea, part 1: a status report on the disease state, general measures, and adjunctive skin care. Cutis. 2013;92:234-40.

11. Erdogan HK, Bulur I, Saracoglu ZN, et al. The evaluation of contact sensitivity with standard and cosmetic patch test series in rosacea patients. Ann Dermatol. 2018;30:290-5.

12. Chang Y-S, Huang Y-C. Role of Demodex mite infestation in rosacea: a systematic review and meta-analysis. J Am Acad Dermatol. 2017;77:441-7. 